\title{
Assessing the performance of biogas plants with multi-criteria and data envelopment analysis
}

\author{
Reinhard Madlener ${ }^{\mathrm{a}, *}$, Carlos Henggeler Antunes ${ }^{\mathrm{b}}$, Luis C. Dias ${ }^{\mathrm{c}}$ \\ a Institute for Future Energy Consumer Needs and Behavior (FCN), Faculty of Economics/E.ON Energy Research Center, RWTH Aachen University, \\ Templergraben 55, 52056 Aachen, Germany \\ ${ }^{\mathrm{b}}$ Department of Electrical Engineering and Computers, University of Coimbra and INESC Coimbra, Rua Antero de Quental, 199 Coimbra, Portugal \\ ${ }^{\mathrm{c}}$ Faculty of Economics, University of Coimbra and INESC Coimbra, Rua Antero de Quental, 199 Coimbra, Portugal
}

\section{A R T I C L E I N F O}

Article history:

Received 18 January 2007

Accepted 19 December 2007

Available online $\mathrm{xxxx}$

\section{Keywords:}

Multi-criteria decision analysis

DEA

Renewable energy

Biogas

ELECTRE TRI

\begin{abstract}
A B S T R A C T
This paper performs an assessment of 41 agricultural biogas plants located in Austria to determine their relative performance in terms of economic, environmental, and social criteria and corresponding indicators. The comparison of these renewable energy conversion plants is based on two complementary analyses. Data envelopment analysis (DEA) was conducted to provide measures of radial efficiency relative to the observed frontier of production possibilities. Multi-criteria decision analysis (MCDA), using the IRIS/ ELECTRE TRI methodology, was conducted to obtain a different perspective on the results, and as a tool that would enable to incorporate managerial preferences easily. To be able to use IRIS while keeping the spirit behind DEA, the evaluation criteria were defined as different output/input efficiency ratios, and no information about criteria weights was introduced at the outset. The results suggest that MCDA, and the use of IRIS in particular, constitutes a useful approach that can be applied in a complementary way to DEA.
\end{abstract}

(c) 2008 Elsevier B.V. All rights reserved.

\section{Introduction}

Over the last two decades, a growing environmental awareness has changed the focus of energy planning processes from an almost exclusive concern with cost minimization of supply-side options to the need of explicitly including multiple and potentially conflicting aspects, such as cost and environmental issues, in decision support models. It is now widely recognized that the largest source of atmospheric pollution stems from fossil fuel combustion, upon which current energy production and use patterns throughout the world rely heavily. Therefore, severe environmental problems arise from energy demand to sustain human needs and economic growth. A more intensive use of renewable energy sources (RES) by means of modern energy conversion technologies can be an important remedy. Although the effective potential of RES is far from being fully exploited, they are becoming increasingly important as supply-side options to satisfy energy needs, taking into account their dispersed generation capabilities, low levels or absence of pollutant emissions, and waste valuation potential. However, some drawbacks can also be associated with RES, such as their intermittent nature, as in the case of wind turbines, and various

\footnotetext{
* Corresponding author. Tel.: +49 2418097 162; fax: +49 2418092206.

E-mail addresses: rmadlener@eonerc.rwth-aachen.de (R. Madlener), cantunes@ inescc.pt (C.H. Antunes), ldias@inescc.pt (L.C. Dias).
}

types of negative environmental impacts (Abbasi and Abbasi, 2000; Dincer and Rosen, 1999).

The Kyoto Protocol, the EU Renewables Directive 2001/77/EC, and the European Biomass Action Plan are examples of ambitious political goals fostering the development of energy conversion technologies based on RES. In this paper we address the case of agricultural biogas plants in Austria, which use mainly energy crops (silage) for anaerobic digestion, that have been effectively promoted over the last couple of years through investment subsidies (capital grants) and, probably more importantly, also by means of guaranteed feed-in tariffs for electricity sold to the grid.

From an interdisciplinary point of view, the assessment of the global performance of different entities (potential solutions, courses of action, decision alternatives) can no longer be based on a single-dimensional axis of evaluation, such as cost or benefit. In most cases, multiple, incommensurate, and often conflicting axes of evaluation of distinct nature are inherently at stake. Therefore, economic, technical, societal, and environmental aspects must be explicitly taken into account in models for decision support, rather than aggregated in a single (and typically economic) indicator.

This paper uses both data envelopment analysis (DEA) and multi-criteria decision analysis (MCDA) approaches for assessing the efficiency of 41 agricultural biogas plants, with the purpose of gaining some new insights about combining these complementary evaluation techniques as well as the underlying methodologies. On 
the one hand, DEA is the tool generally used to evaluate the efficiency of decision making units (DMUs). These are comparable organizational entities performing similar tasks in a homogeneous operating environment. On the other hand, MCDA is the tool generally used to conciliate multiple evaluation criteria, taking into account the preferences of a decision-maker. The introduction of managerial preference information is often relevant when assessing the relative performance of the DMUs. In fact, a manager is normally not indifferent as to whether a unit turns out to be efficient by using a less important combination of inputs and/or outputs, and by underweighting inputs and/or outputs of high importance to the business concerned.

With these intentions in mind, this paper also addresses the challenge of determining how MCDA methods can be used in the context of efficiency evaluation, trying to keep the spirit behind DEA, while being able to use MCDA's capabilities of explicitly incorporating the preferences of a decision-maker, not necessarily in the form of trade-off restrictions.

Uncertainty is an intrinsic characteristic of real-world problems arising from multiple sources of distinct nature. Uncertainty emerges from the ever-increasing complexity of interactions within social, economic environmental and technical systems, characterized by a fast pace of technological evolution, changes in market structures, and new societal concerns. It is generally impracticable to envisage decision aid models that would capture all the relevant interrelated phenomena at stake, incorporate and process all the necessary information, and also account for the changes and/or hesitations associated with the explicit expression of the stakeholders' preferences. Besides structural uncertainty associated with the global knowledge about the system being modeled, input data may also suffer from imprecision, incompleteness, or may be subject to changes. In this context, it is important to provide managers and decision-makers with robust conclusions (Roy, 1998; Vincke, 1999). The concept of a robust solution is generally linked to (1) a certain degree of "immunity" to data uncertainty, (2) an adaptive capability (or flexibility) regarding an uncertain future or ill-specified preferences, and (3) the guaranteeing of an acceptable performance even under changing conditions (drifting from "nominal data"). This motivated the choice of the IRIS/ELECTRE TRI methodology, which is fairly robust to changes in data, is able to cope with imprecisely defined preferences, and produces only a partitioning of the DMUs into classes, rather than a complete ranking of the DMUs.

The paper is organized as follows: Section 2 introduces and compares the two analytical frameworks studied. Section 3 describes the case study and how DEA and MCDA have been applied. The main results obtained are reported in Section 4. In Section 5 the findings from the analysis are discussed and some conclusions are drawn.

\section{Comparison of analytical frameworks}

\section{1. $D E A$}

The attainment of high levels of performance is a key issue for the success of every organization. Therefore, an adequate management framework is necessary for evaluating the current performance, identifying benchmarks to use in seeking improvements, and understanding why some units in a particular organization are operating (in-)efficiently.

DEA is a non-parametric performance measurement technique, based on linear programming (LP), for assessing the efficiency of DMUs (e.g., Charnes et al., 1985; Cooper et al., 2000) relative to an observed set of production possibilities. DMUs are homogeneous entities (such as sales outlets, electricity distribution compa- nies, bank branches, schools, university departments, etc.) with some decision autonomy, operating a production process that converts a set of inputs into a set of outputs. DEA models use these inputs and outputs to compute an efficiency score for a given DMU when this particular DMU is compared with all the other DMUs considered. The relative efficiency of a DMU is usually defined as the ratio between the sum of its weighted output levels to the sum of its weighted input levels. The weights are chosen by the LP model such that a DMU is "shown in its best light", i.e., that its efficiency score is maximized. In contrast to parametric econometric approaches, such as stochastic frontier analysis, DEA does not assume any specific functional form, thus avoiding problems of model misspecification.

In DEA, a DMU is considered efficient if there is no other DMU, or a linear combination of inputs and outputs of several DMUs, that can improve one input or output, without worsening the value of at least another one. The frontier is defined by the observed values of the (relatively) efficient DMUs. If a DMU does not belong to this envelopment surface and lies in its interior, then that DMU is operating inefficiently. DEA models usually return an efficient projection point of operation on the frontier for each inefficient DMU, thus identifying the DMUs that can be used as performance benchmarks (the "peers") for the DMUs that are operating inefficiently.

Three basic DEA models are generally distinguished: CCR model, BCC model, and Additive model (see Cooper et al., 2000, 2004; for a presentation and comparative analysis of these models). The CCR model was presented in the seminal work of Charnes et al. (1978). It is based on the radial minimization (maximization) of all inputs (outputs) and assumes an environment of constant returns to scale (CRS), i.e., if an efficient DMU increased its inputs by a factor of $\alpha$, then its outputs would be expected to increase by the same factor.

Let us consider $n$ DMUs to be evaluated; each of them consumes $m$ inputs to produce $p$ outputs. The input data is denoted by the matrix $\mathbf{X}_{m \times n}$ and the output data is denoted by the matrix $\mathbf{Y}_{p \times n}$. We denote by $X_{j}$ (the $j$ th column of $\mathbf{X}$ ) the vector of inputs consumed by DMUj, and by $x_{i j}$ (a positive quantity) the quantity of input $i$ consumed by DMUj. Analogous notation $Y_{j}$ and $y_{i j}$ is used for outputs. We denote by 1 the summation vector $(1, \ldots, 1)$.

The envelopment linear programming formulation to assess the efficiency of a DMU $\left(X_{k}, Y_{k}\right)$ and its dual, the multiplier formulation, for the (input-oriented) CCR model can be written as

$$
\begin{array}{llll}
\min & \theta-\varepsilon(\mathbf{1} s+\mathbf{1} e) & \max & \mu Y_{k} \\
\text { s.t. } & \mathbf{Y} \lambda-s=Y_{k}, & \text { s.t. } & v X_{k}=1, \\
& \theta X_{k}-\mathbf{X} \lambda-e=0, & & \mu \mathbf{Y}-v \mathbf{X} \leqslant 0, \\
& \lambda \geqslant 0, & & v \geqslant \varepsilon \mathbf{1}, \\
& e \geqslant 0, s \geqslant 0 . & & \mu \geqslant \varepsilon \mathbf{1} .
\end{array}
$$

DMU $\left(X_{k}, Y_{k}\right)$ is considered efficient only if the optimal solution of the envelopment formulation yields $\theta=1$ (the radial efficiency measure) and all "slacks" are null (i.e., $s=0$ and $e=0$ ). The multiplier formulation emphasizes the relative weight vectors chosen by the DMU for the inputs $(v)$ and the outputs $(\mu)$. A very small constant $\varepsilon$ prevents null weights.

For the cases where the constant returns to scale assumption is dropped, Banker et al. (1984) proposed a variable returns to scale (VRS) version of the CCR model, referred to as the BCC model. The difference between the two types of envelopment surfaces, CRS and VRS, is that the latter is subject to a "convexity constraint" so that the set of production possibilities is defined as the set of convex combinations of the observed DMUs. In the envelopment form of the linear programming formulation, this amounts to adding the constraint $\mathbf{1} \lambda=1$. The third type of DEA model, the Additive model (Charnes et al., 1985), also assumes VRS but is less frequently applied. 
DEA models have been extensively used to assess the performance of DMUs in a broad range of real-world problems. However, some important issues regarding the application of DEA with realworld data remain. Firstly, the complete weight flexibility assumed by DEA models often leads to efficiency results that are difficult to justify. The freedom of each DMU to choose the weights of inputs and outputs that show it under the best possible light can lead to the assignment of very low weights to some inputs or outputs. In practice, this means that certain inputs or outputs are effectively ignored (a disturbing effect of the free specialization allowed in DEA models, which is not generally acceptable in practice).

Moreover, the inputs and outputs can be weighted in a manner that contradicts the views and/or preferences of the organizations and their stakeholders, or even in a quite counterintuitive manner by valuing secondary inputs or outputs more than priority ones (Joro and Viitala, 2004). In fact, the inputs and outputs are not generally equally relevant and some preference information must be included in the analysis. Also, whenever the number of inputs and outputs grows, the trend is that more DMUs become efficient, thus impoverishing the discriminating power of the DEA models.

One of the techniques generally used to circumvent these issues is the introduction of additional restrictions on the variation allowed for the weights. The most common type of weight restrictions (for a review see Thanassoulis et al., 2004) are assurance regions of type I (intra inputs or intra outputs) or of type II (relating inputs to outputs); other variants include transforming the data matrices, or adding fictitious (unobserved) DMUs. However, as pointed out by some authors (e.g., Podinovski, 2004), the resulting efficiency score of weight-restricted models cannot be interpreted as a realistic improvement factor (because the efficient radial target of an inefficient DMU is no longer technologically feasible). Furthermore, these approaches are not natural to capture scaleindependent subjective value judgments elicited from the managers on the perceived importance of inputs and outputs: they are more appropriate to reflect objective information such as prices. However, in real-world problems in which inputs and outputs are less tangible, market costs and prices may not be readily available, which introduces an additional degree of arbitrariness to the results.

\section{2. $M C D A$}

The above-mentioned considerations about DEA led us to envisage the use of MCDA models to perform efficiency evaluation. MCDA (see, e.g., Roy, 1996) includes a variety of sound theoretical frameworks for eliciting and representing preferences. Some of these frameworks, such as the one suggested in this paper, require weights that do not necessarily represent prices or re-scaling coefficients.

Instead of attempting to assign an efficiency measure to each DMU we believe that, in most real-world situations, assigning the DMUs to ordered efficiency categories is sufficient for analysis and provides more confidence about the results, in the sense of robustness to changes either in data or managers' preferences, than a single numerical figure. Moreover, a more detailed analysis with- in each efficiency category is always possible whenever it is found useful to improve the discrimination of the evaluation model.

In assessing the performance of DMUs in which technical, economic and environmental aspects are at stake, it is often important to use known standards (or theoretical maxima) and efficiency profiles. There are also situations in which DMUs must be appraised for efficiency on an "as they come" basis, i.e., they are not included in a given set of DMUs (e.g., in growing markets, where more and more DMUs are established over time). This required capability of evaluating each DMU in absolute terms, and not just in comparison with other peers, as well as the need to include evaluation aspects expressed in different units, using any sort of scales (including qualitative), can be achieved using the ELECTRE TRI method (Yu, 1992).

The ELECTRE TRI method belongs to the ELECTRE family of multi-criteria methods developed by Bernard Roy and his co-workers (Roy, 1996). ELECTRE methods are based on the construction and exploitation of a so-called outranking relation ("outranking" in this context means "is at least as good as"). ELECTRE TRI is devoted to the sorting problem (in contrast to choice or ranking problems), which consists in partitioning a set of entities being evaluated into a pre-defined set of ordered categories, according to several evaluation criteria. Each entity object of evaluation (DMUs, in DEA language, or "action", in ELECTRE language) is described through a vector of multi-criteria performances. The categories $\left(C^{1}, \ldots, C^{k}\right)$ are also defined by specifying multi-criteria performance vectors $\left(b^{0}, \ldots, b^{k}\right)$, or reference profiles. Each reference profile $b^{h}(h=1, \ldots, k-1)$ is simultaneously the upper bound of category $C^{h}$ and the lower bound of category $C^{h+1}$ (see Fig. 1).

The assignment of each entity $a$ to a category $C^{h}$ is done by comparing its value in each criterion to the reference profiles. The procedure assigns each entity to the highest category such that its lower bound is outranked by $a$. The outranking relation is verified by comparing a credibility index, computed by using the differences in performance and the criterion weights, with a cutting level $\lambda(\lambda \in[0.5,1])$, which defines the "majority requirement" and hence the exigency of the classification. For further details about ELECTRE TRI see Yu (1992) and Mousseau et al. (2000), among others.

Multi-criteria methods usually require a set of parameters that embody the preferences of the decision-makers. The ELECTRE TRI method requires the specification of the reference profiles associated with the categories $\left(b^{0}, \ldots, b^{k}\right)$, the criterion weights, and the cutting level $(\lambda)$. Also, a set of indifference $\left(q_{j}\right)$, preference $\left(p_{j}\right)$ and veto $\left(v_{j}\right)$ thresholds for each criterion $j$ and reference profiles can be defined. Indifference and preference thresholds characterize the acceptance of imprecision in the judgment by considering two entities as indifferent when their individual performances in each criterion $j$ differ less than a specified amount $q_{j}$. Moreover, the transition from indifference to preference is made gradual, changing linearly from $q_{j}$ to $p_{j}$. The veto thresholds are aimed at capturing situations in which very bad scores in any criterion should prevent an entity of being classified in the best category, or if these bad scores should force it to be classified in the worst category independently of having very good scores in all other criteria. This

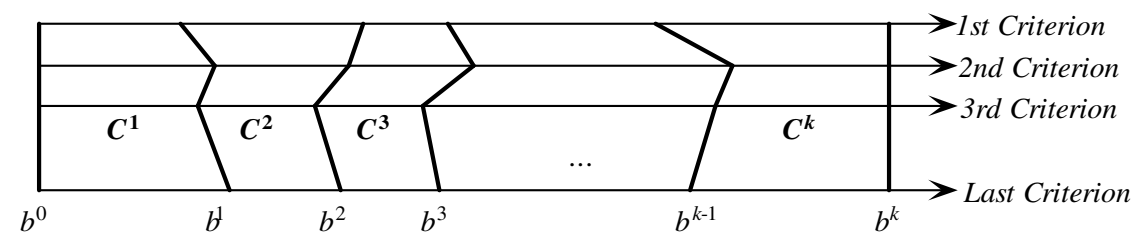

Fig. 1. Definition of categories $C^{1}, \ldots, C^{k}$ through reference profiles $b^{0}, \ldots, b^{k}$. 
enables, as it is often required in practice, to introduce a certain level of non-compensation into the evaluation model.

In the present application of dealing with an efficiency evaluation problem by means of a multi-criteria sorting model the software IRIS has been used (Dias and Mousseau, 2003). IRIS implements a methodology developed by Dias et al. (2002) that is based on the ELECTRE TRI method, but which does not require precise values for some input parameters (criteria weights and the cutting level). Information about these parameter values can be provided through the introduction of intervals, linear constraints, or even sorting examples (which are translated into constraints on the parameters that guarantee that those example results are reproduced). Given these constraints on the parameter values, IRIS infers a "central" vector through the maximization of the minimum slack associated with the constraints, when the constraints are consistent. For each entity being evaluated, IRIS shows the category corresponding to this central combination, and the other possible classifications that respect the constraints imposed. In case the set of constraints is inconsistent, IRIS suggests the constraint subsets that may be removed to restore consistency.

A relevant issue in this context is the meaning of the weights in ELECTRE methods. In this type of methods, weights are perceived as true coefficients of importance assigned to the criteria, and not just as technical devices for translating the performances in the criteria considered into a common value measure. Therefore, they are scale-independent (that is, they are not linked to the scales in which each criterion is measured), thus making them easier to be specified by managers. These parameters bear the preference information and insights into the sorting process. In principle, they must be elicited from managers and stakeholders (preferably via an analyst with expertise on the methodological component). It should be noted that this method imposes a non-negligible burden associated with the specification of all the parameters required. However, some of these parameter data can be preset according to the experience of the analyst, in general associated with previous case studies. For instance, indifference and preference thresholds can be fixed as percentages (say $1 \%$ and $10 \%$, respectively) of the value ranges in each category.

The IRIS software allows for the consideration of uncertainty in the weights (as well as in the cutting level). This feature contributes to reducing the data requirements and increasing the confidence in the results.

\section{Case study}

In Austria, an effective promotion of renewable energy technologies has been pursued in recent years, driven by the need to achieve ambitious energy and climate policy goals. These include the goals contained in the Kyoto Protocol $(-13 \%$ greenhouse gas emissions by 2008/12, relative to 1990 levels) and the EU Renewables Directive 2001/77/EC (to raise the renewable electricity share of Austria to $78.1 \%$ by 2008 , compared to $70 \%$ in 1997). In particular, the last few years witnessed a remarkable boom in the construction of agricultural biogas plants, mainly due to the introduction of substantial feed-in tariffs of between 10.3 and 16.5 Cents $€ / \mathrm{kWh}_{\mathrm{el}}$ (depending on the plant size and the type of substrate used) for "biogas electricity" fed into the grid, which are guaranteed for a period of 13 years (Green Electricity Act, 2002). ${ }^{1}$ As a consequence, the number of plants rose from 119 at the end of 2003 to 231 by the end of year 2005 (Braun et al., 2007). These plants use mainly energy crops (silage) for digestion.

\footnotetext{
${ }^{1}$ Note that in 2006 a revised Green Electricity Act and Ordinance entered into force, with amended feed-in tariffs and budget restrictions (BGB1. I Nr. 105/2006, BGBl. II Nr. 401/2006; for details see Energie-Control, 2006).
}

However, up to now the promotion of energy crop digestion was hardly linked to any specific cost effectiveness or energy efficiency or other performance criteria. As a result, many different technologies, design concepts, and specific applications occurred on the market, some of which were either not very productive, energy-efficient, or reliable.

Due to the attractive feed-in tariffs granted, anaerobic digestion of energy crops currently mainly aims at the generation of electricity, and much less so at heat generation (or the feed-in of purified biogas into the natural gas grid, if available). As a consequence, the heat energy produced in cogeneration units remains largely wasted. Furthermore, many plants use electricity for cooling purposes, in order to prevent adverse effects that arise from the selfheating of crop digesters. Therefore, in many cases up to two thirds of the available technical energy potential remains unused (Braun et al., 2005, 2007; Walla, 2005).

A comprehensive monitoring and benchmarking project was initiated in March 2004, which includes a detailed investigation of a set of 41 Austrian energy crop digestion plants spread all over the country. ${ }^{2}$ The project, completed in February 2007, also aimed at creating and establishing an evaluation system for the transparent assessment and benchmarking of the productivity of biogas plants by means of energetic, business economic, ecological and socio-economic criteria, characterizing the overall production cycle of biogas. Since anaerobic digestion has the potential of reducing greenhouse gas emissions (Braschkat et al., 2003), an important objective of the project was to evaluate the environmental impacts through the overall "crops to energy" process. Finally, positive and negative socio-economic impacts were accounted for to a limited extent by means of a questionnaire survey among plant operators (subjective valuation by the farmers interviewed, supplemented by measurable data); see Braun et al. (2007) for further details.

\section{Results}

\subsection{Description of the data and parameters used}

The DMUs considered are a representative set of energy crop digestion plants in Austria, aimed at covering the whole spectrum of existing plant types and operating conditions. Samples were taken from the substrate, digester, fermentation residues and biogas plant types. Cooling, safe transport and appropriate storage were also scrutinized. The sampled installations are geographically well distributed over the country. They range from small-scale installations using mainly manure and energy crops (down to $18 \mathrm{~kW}_{\mathrm{el}}$ ) to larger-scale plants that use considerable amounts of co-substrates (up to $1.7 \mathrm{MW}_{\mathrm{el}}$ ). Both single substrate (energy crops or manure) installations as well as co-digestion plants (agricultural by-products and industrial bio-wastes) have been considered in the analysis.

The main groups of evaluation aspects at stake for assessing the efficiency of energy crop digestion plants are: (1) substrate provision, storage and pre-treatment; (2) biogas production (by means of anaerobic digestion); (3) net utilization of heat and electricity; (4) digestate handling and disposal; and (5) greenhouse gas (GHG) emissions. ${ }^{3}$

\footnotetext{
2 For a description of the project "Development of an Assessment System for Biogas Plants - 'Quality Certificate Biogas" (funded by the Austrian Federal Ministry of Transport, Innovation and Technology) see http://www.energiesystemederzukunft.at/ results.html/id3469?active= (in German), or research database entry \#8289 in www.rdb.ethz.ch (in English).

${ }^{3}$ Based on life cycle analysis, a biogas plant may or may not reduce greenhouse gas emissions, compared to a situation where the plant does not exist. The net amount of GHG emissions attributable to a particular biogas plant depends, in essence, on the type of fertilizer used, the fossil fuel use for substrate production and transporting, and the methane emissions released from the digested substrate storage facility, the spreading of digested substrate in the fields, and the cogeneration unit
} 
Table 1

Descriptive statistics $(N=41)$

\begin{tabular}{lrrrr}
\hline & \multicolumn{1}{l}{ Mean } & \multicolumn{1}{l}{ SD } & Min & \multicolumn{1}{c}{ Max } \\
\hline $\begin{array}{l}\text { Inputs } \\
i_{1}-\text { labor }\end{array}$ & 1581.29 & 1958.51 & 50.42 & 10950.00 \\
$i_{2}-$ ODS & 1508.00 & 1333.75 & 119.94 & 5514.04 \\
Outputs & & & & \\
$o_{1}-$ electricity & 1940136.06 & 1960911.08 & 123600.83 & 7760000.00 \\
$o_{2}-$ heat & 735319.76 & 1112935.01 & 0.00 & 6000000.00 \\
$o_{3}-$ GHG (undesirable & 248532.63 & 282431.82 & -203248.11 & 1123981.23 \\
\multicolumn{1}{c}{ output) } & & & & \\
\hline
\end{tabular}

In a first series of model specifications, the following criteria have been considered for evaluating the efficiency of the energy crop digestion plants (for the sake of comparison between the DEA and the MCDA approaches): (1) labor (i.e., time) spent for plant operation; (2) amount of substrate used (organic dry substance, ODS); (3) amount of biogas or net electricity produced (i.e., electricity delivered by the biogas plant for external consumption, net of what the plant consumes itself); (4) net heat produced (for external consumption); and (5) net GHG emissions released to the atmosphere (including credits that accrue from a comparison with the base case of not having the biogas plant, measured in $\mathrm{CO}_{2}$ equivalent). For further details on data collection see Braun et al. $(2005,2007)$ and Laaber et al. (2005), and for further details about the various inputs and outputs and DEA model specifications scrutinized see Madlener (2006). Some descriptive statistics are displayed in Table 1.

\section{2. $D E A$}

In the first DEA model considered in this paper, we have used substrate $\left(i_{2}\right)$ and labor $\left(i_{1}\right)$ as inputs and the amount of net electricity $\left(o_{1}\right)$ and external heat $\left(o_{2}\right)$ as (desirable) outputs. GHG emissions $\left(\mathrm{O}_{3}\right)$ have been considered as well in a second model, as an undesirable output. We chose to consider these emissions as an input in the DEA model, which is a common option to model undesirable outputs. Although other options exist (e.g., see Scheel, 2001; Seiford and Zhu, 2002), and although the choice influences the results, there does not seem to be an undisputed ideal method to handle undesirable outputs (Dyson et al., 2001). A scale transla- tion was used to account for the negative net values, another modeling option known to have an influence on the results (Lovell and Pastor, 1995). Fig. 2 depicts the outcome of the (output-oriented) CCR DEA model specification (CCR-O). DMUs 12, 17, 18, 20 and 28 form the efficiency frontier.

A similar analysis was performed considering GHG emissions. These results are depicted in Fig. 3. The main consequence of incorporating this new factor into the analysis is that DMU 15, which has relatively low net emissions, joins the set of efficient solutions. On the other end of the spectrum, DMUs 5, 13, 14, 25, 26, 33, and 39 appear as some of the worst-performing plants, irrespective of whether GHG emissions are considered or not.

The DEA results just reported are part of a wider study (Braun et al., 2007; Madlener and Honegger, 2006) where other models were considered as well (e.g., considering the output Biogas instead of Electricity and Heat), including versions using the BCC model to benefit DMUs not operating at an optimal scale. In this paper, however, we will consider only the results presented in Figs. 2 and 3, with the underlying assumption of constant returns to scale, since the MCDA approach described below will also not take into account the scale of operation. Keeping the same inputs and outputs presented here, the main implications of using the variable returns to scale version (BCC-O) would be to add DMU 15 to the set of efficient units, without GHG emissions, and adding DMUs 33 and 38 to the set of efficient units, with GHG emissions considered.

\section{3. $M C D A$}

In the MCDA approach the objective was to identify groups of DMUs that could be assigned to different efficiency labels, rather than computing a precise efficiency score or deriving a complete ranking. Four efficiency categories were defined to classify the DMUs according to their efficiency: $C^{1}=$ "Poor", $C^{2}=$ "Fair", $C^{3}=$ "Good", and $C^{4}=$ "Very good". Each plant has to be assigned to one of these ordered categories, according to the multiple evaluation criteria.

To define the different categories in IRIS, it is necessary to set the category bounds $b^{0}, \ldots, b^{4}$ according to $n_{\text {crit }}$ criteria/indicators which we denote as the evaluation functions $g_{j}(\cdot)\left(j=1, \ldots, n_{\text {crit }}\right)$. The decision-maker must set these bounds, taking into account that according to an indicator $g_{i}(\cdot)$ a $\mathrm{DMU}_{k}$ with $g_{i}\left(\mathrm{DMU}_{k}\right) \in\left[g_{i}\left(b^{j-1}\right), g_{i}\left(b^{j}\right)\left[\right.\right.$ should be placed into category $C^{j}$. When

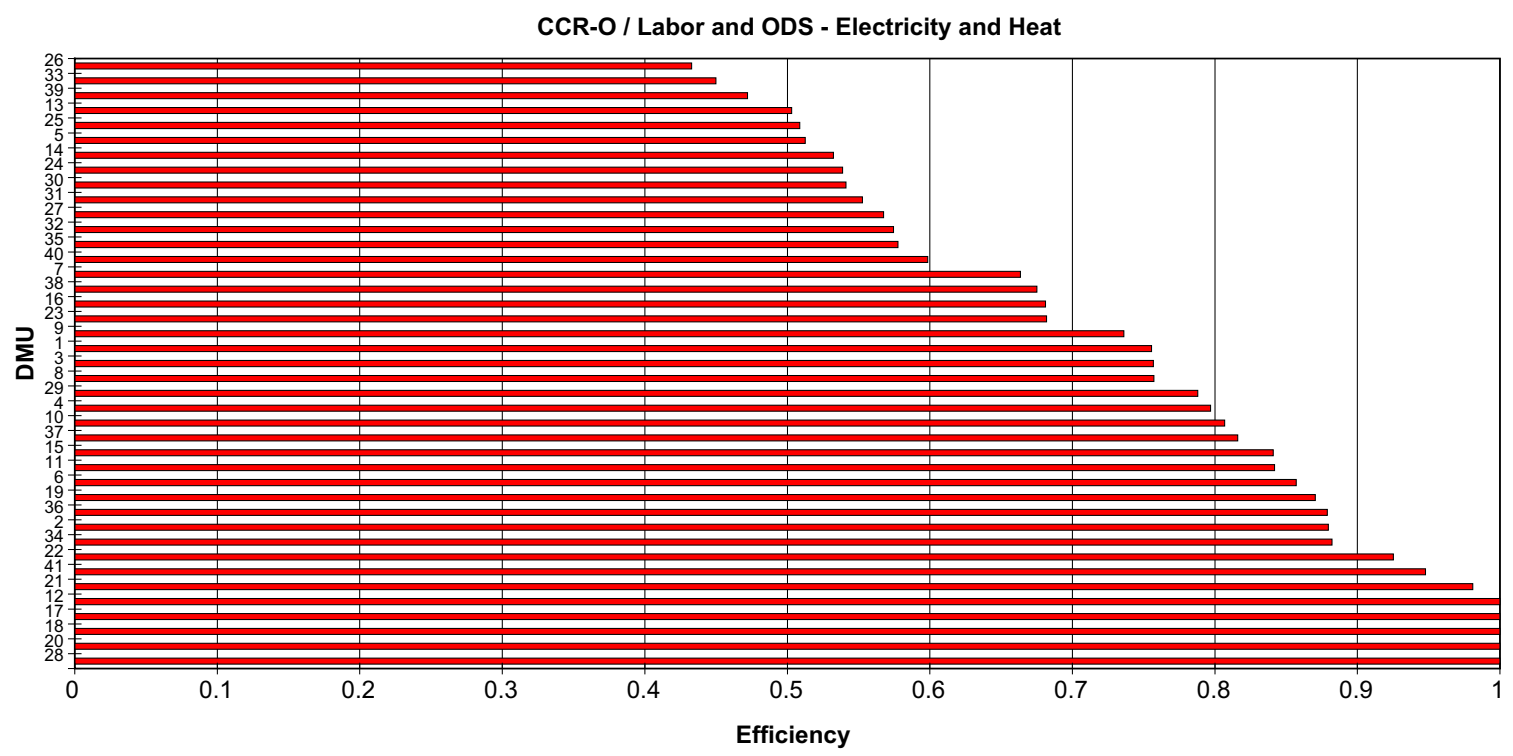

Fig. 2. DEA results without GHG emissions (inputs: labor, substrate; outputs: electricity, heat). 


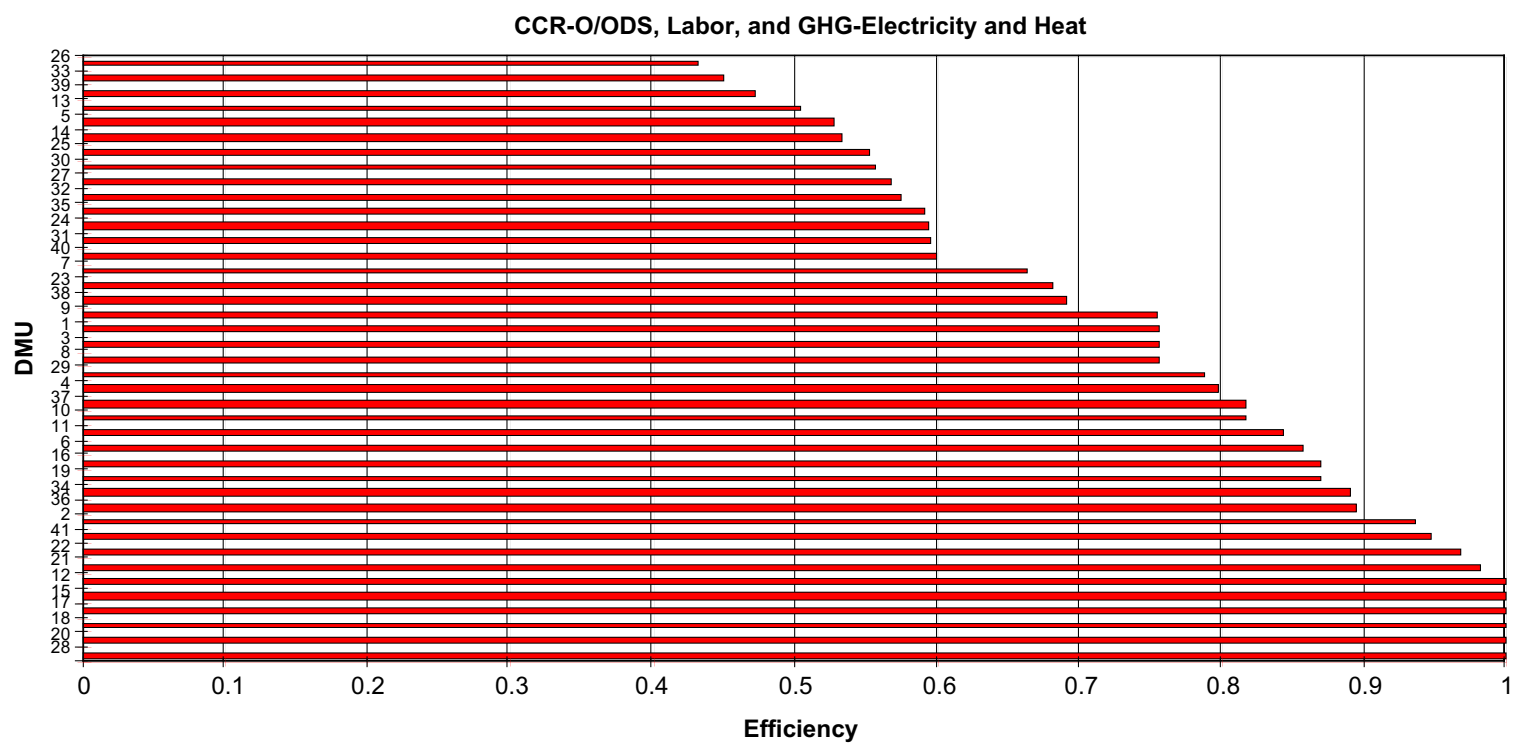

Fig. 3. DEA results when including GHG emissions (inputs: labor, substrate, GHG; outputs: electricity, heat).

attempting to set these parameters, it became clear that the inputs and outputs from the DEA could not be taken as sorting criteria without some kind of adaptation. For instance, in which efficiency category should a DMU with electricity production (indicator $o_{1}$ ) of 900000 be sorted? Clearly, such questions concerning the efficiency of a specific plant cannot be answered without knowing at least the dimension of the plant or the inputs it consumes.

One of the modeling options would be to consider as sorting indicators the ratio of inputs and outputs regarding some surrogate for the dimension of the plant, e.g., the amount of organic dry substrate (ODS) used. The indicators would then be labor/ODS $\left(i_{1} / i_{2}\right)$, electricity/ODS $\left(o_{1} / i_{2}\right)$, heat/ODS $\left(o_{2} / i_{2}\right)$, and GHG/ODS $\left(o_{3} / i_{2}\right)$. Another option, which we have used in the experiments described below, is to use all ratios between the outputs and the inputs in the DEA model: the multiple indicators are ratios combining an output to maximize or minimize (in the numerator) with an input (in the denominator). Thus, the following indicators have been considered: electricity/labor $g_{1}=o_{1} / i_{1}(\max )$, electricity $/$ ODS $g_{2}=o_{1} / i_{2}$ (max), heat/labor $g_{3}=o_{2} / i_{1}(\max )$, heat/ODS $g_{4}=o_{2} / i_{2} \quad(\max )$, GHG/labor $g_{5}=o_{3} / i_{1}(\mathrm{~min})$, and GHG/ODS $g_{6}=o_{3} / i_{2}(\mathrm{~min})$. The corresponding descriptive statistics are displayed in Table 2 . Note that although this approach leads to a high number of indicators as the number of criteria increases, it mimics the spirit of DEA: to allow each DMU to be evaluated according to multiple indicators and to choose the most favorable indicators (within the constraints that the decision-maker may impose, as we will illustrate further below). It would also be possible to consider indicators electricity/GHG $\left(o_{1} / o_{3}\right)$ and heat/GHG $\left(o_{2} / o_{3}\right)$, both to be maximized, for

Table 2

Descriptive statistics for the MCDA indicators (41 DMUs)

\begin{tabular}{lrrrr}
\hline & \multicolumn{1}{c}{ Mean } & \multicolumn{1}{c}{ SD } & \multicolumn{1}{c}{ Min } & \multicolumn{1}{c}{ Max } \\
\hline To maximize & & & & \\
$g_{1}$ - electricity/labor & 1803.49 & 2110.86 & 215.41 & 12814.42 \\
$g_{2}$ - electricity/ODS & 1164.21 & 280.74 & 666.98 & 1874.74 \\
$g_{3}$ - heat/labor & 641.20 & 666.02 & 0.00 & 2935.54 \\
$g_{4}$ - heat/ODS & 593.39 & 531.00 & 0.00 & 2308.08 \\
To minimize & & & & \\
$g_{5}-\mathrm{GHG}^{\mathrm{a}} /$ labor & 1998.05 & 3079.48 & 147.80 & 19948.65 \\
$g_{6}-\mathrm{GHG}^{\mathrm{a}}$ /ODS & 1696.94 & 1665.57 & 301.43 & 8385.64 \\
\hline
\end{tabular}

a The same scale translation as the one applied in DEA was used. replacing $g_{5}$ and $g_{6}$, respectively, since $o_{3}$ is an undesirable output. Although this would be closer to the DEA models, it was felt that $g_{5}$ and $g_{6}$ reflected better the perspective of an external evaluation body responsible for assigning the plants to efficiency categories or labels.

The reference profiles, which define the limits between each category, were defined such that approximately $1 / 4$ of the DMUs are placed in each category according to each indicator individually (see Table 3 ). Hence, for each indicator $g_{j}$ and for each category $C^{h}$, there are approximately 10 plants that would be sorted into that category according to the indicator concerned.

Fig. 4 presents some conclusions that may be drawn without making any distinction between the relative importance of each indicator. An "Optimistic" classification corresponds to what would result if each DMU was allowed to choose one indicator, i.e., if the DMU was allowed to specify the ELECTRE TRI weights of the indicators, setting one of them to have a weight equal to 1 and all the remaining indicators as having null weight. These are the classifications that are more in accordance with the spirit behind DEA, but are not very discriminative when only four categories are used: 28 DMUs would be considered as "Very good" $\left(C^{4}\right)$ and the remaining 13 DMUs would be considered as "Good" $\left(C^{3}\right)$. A "Pessimistic" classification indicates the worst category suggested by some indicator. This means that a DMU is classified into category $C^{h}$ if and only if all the six indicators place it in that category (or in a better one). According to this classification, most of the DMUs would be considered as "Poor", six of them would be considered as "Fair", three of them would be considered as "Good", and only one (DMU 20) would be considered as "Very good". An intermediate classification ("50\% majority") indicates what would result if we required the support of at least half of the indicators: a DMU is classified into category $C^{h}$ if and only if three out of six indicators place it in that category (or in a better one). In this (more discriminating) case, 11 DMUs would be considered as "Very good", 13 DMUs would be considered as "Good", 15 DMUs would be considered as "Fair", and two DMUs (number 30 and 32) would be considered as "Poor".

If the DMUs were entirely free to choose the weights assigned to the indicators, then all DMUs would be sorted into the top two categories, as in this case each DMU is allowed to be judged according to only one of the six indicators, that single indicator being chosen by the DMU. In order to decrease the number of 
Table 3

Category definitions for each indicator

\begin{tabular}{|c|c|c|c|c|c|c|}
\hline Category & $g_{1}(\max )$ electricity/labor & $g_{2}$ (max) electricity/ODS & $g_{3}(\max )$ heat/labor & $g_{4}(\max )$ heat/ODS & $g_{5}(\mathrm{~min}) \mathrm{GHG} /$ labor & $g_{6}(\mathrm{~min}) \mathrm{GHG} / \mathrm{ODS}$ \\
\hline$C^{1}-$ poor & $<580$ & $<960$ & $<150$ & $<130$ & $>2000$ & $>1800$ \\
\hline$c^{2}-$ fair & {$[580,1100[$} & {$[960,1130[$} & {$[150,375[$} & {$[130,530[$} & ] $1200,2000]$ & ] $1300,1800]$ \\
\hline$C^{3}-\operatorname{good}$ & {$[1100,2300[$} & {$[1130,1300[$} & {$[375,950[$} & {$[530,880[$} & ] $675,1200]$ & ] $600,1300]$ \\
\hline$C^{4}$ - very good & $\geqslant 2300$ & $\geqslant 1300$ & $\geqslant 950$ & $\geqslant 880$ & $\leqslant 675$ & $\leqslant 600$ \\
\hline
\end{tabular}

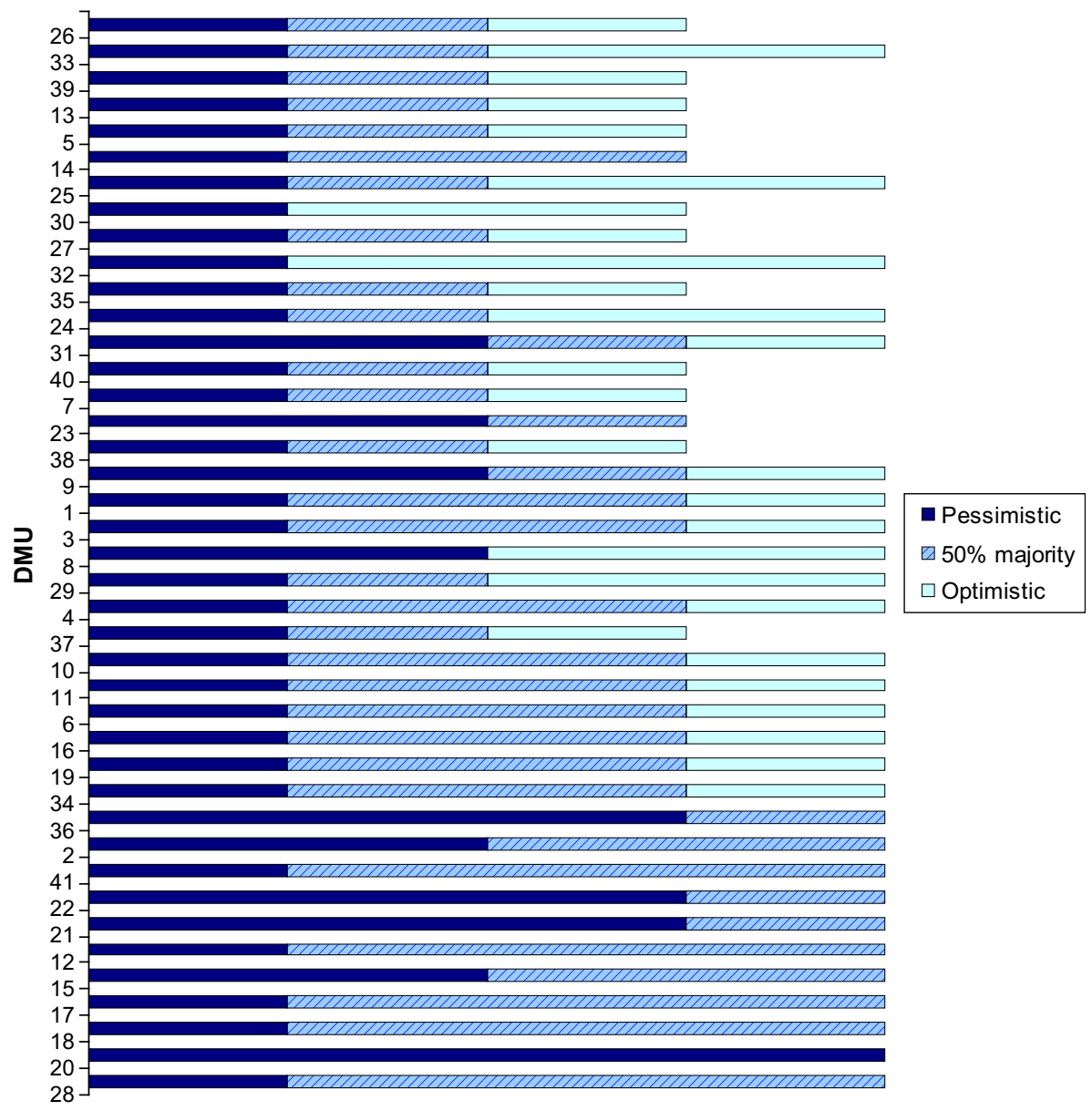

Fig. 4. Pessimistic, 50\% majority, and optimistic classifications (sorted by increasing DEA efficiency score).

DMUs in the best categories, several options can be envisaged: (1) to make the category bound more demanding; (2) to require the support of more than one indicator (e.g., the support of half of the indicators, as depicted in Fig. 4); and/or (3) to add some information about the relative power of the indicators.

\subsection{Comparing the results}

The DMUs in Fig. 4 are ranked by order of their DEA efficiency score (for the case with GHG emissions), from the worst (DMU 26 ) to the best (the last six DMUs - 12, 15, 17, 18, 20, and 28 - have an efficiency score of 1 ). It can be seen that the IRIS classifications tend to improve with the efficiency score, although there are some exceptions. This is not unexpected, as the MCDA analysis is based on very different grounds relatively to the DEA analysis.

A DMU is deemed inefficient by the CCR-O DEA model if there exist other DMUs (or a conical combination of existing DMUs) that yield a higher ratio of weighted outputs over weighted inputs than the inefficient DMU, even when the latter chooses the weights attempting to look as efficient as possible. The six most inefficient plants (featuring efficiency scores below 0.55) are depicted in Fig. 5, which presents two plots: DEA factors on the left and MCDA indicators (criteria) on the right. In both plots the scales are normalized (difference to the worst performance divided by the amplitude of the scale), such that " 0 " corresponds to the worst performance and " 1 " corresponds to the best performance on each scale. The DMUs in Fig. 5 exhibit similar profiles, i.e., small plants performing very well in terms of inputs and emissions, but very poor in terms of electricity and heat outputs. All of these DMUs are considered inefficient in comparison with their peer that has a similar profile (DMU 28, see Fig. 7), which does slightly worse in terms of inputs by a small proportion, but better in terms of outputs produced by a much higher proportion.

Fig. 6 is analogous to Fig. 5, but presents the worst DMUs in the MCDA analysis: the set of DMUs that cannot reach category $C^{4}$ in their optimistic classification (roughly meaning they do not reach the "top 10 " in any of the six ratios considered) and have $50 \%$ or more of the indicators placing them in $C^{1}$ or $C^{2}$. The DMUs in Figs. 5 and 6 have similar profiles with few exceptions, and four of them are among the worst according to both approaches (DMUs 5, 13, 

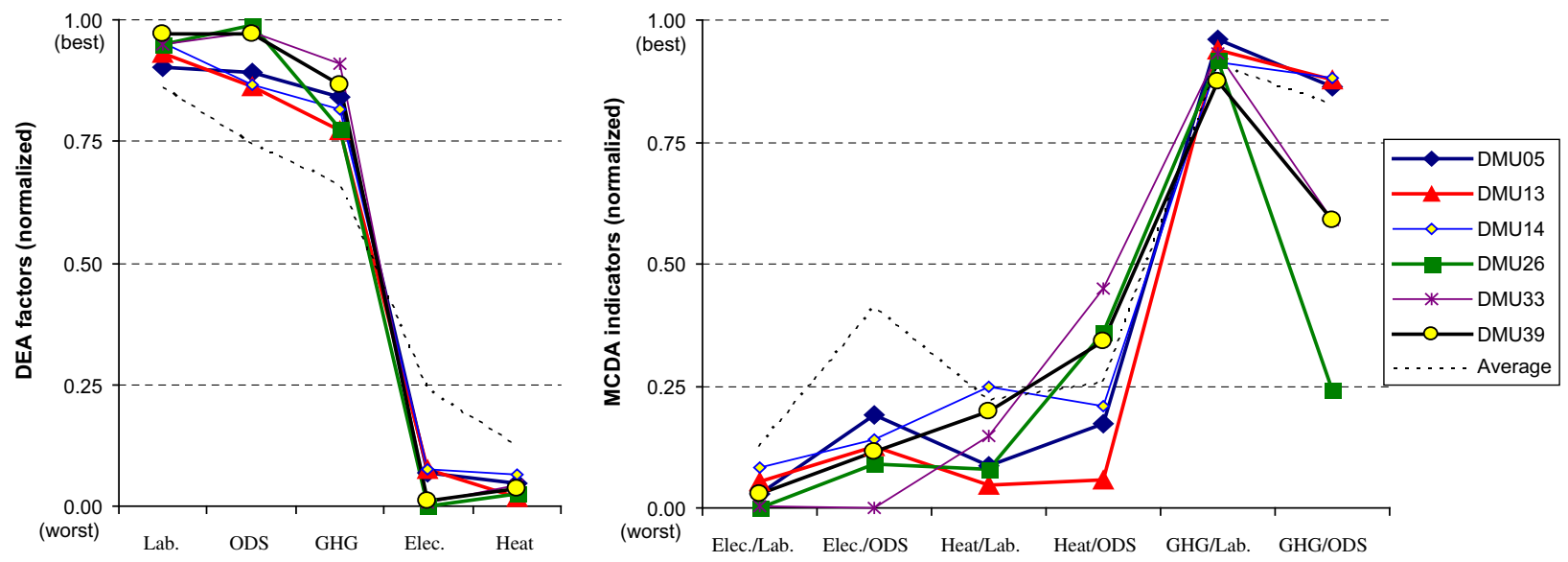

Fig. 5. Normalized DEA factors and MCDA indicators for the worst DMUs according to DEA (DMUs with efficiency scores $<0.55$ ).
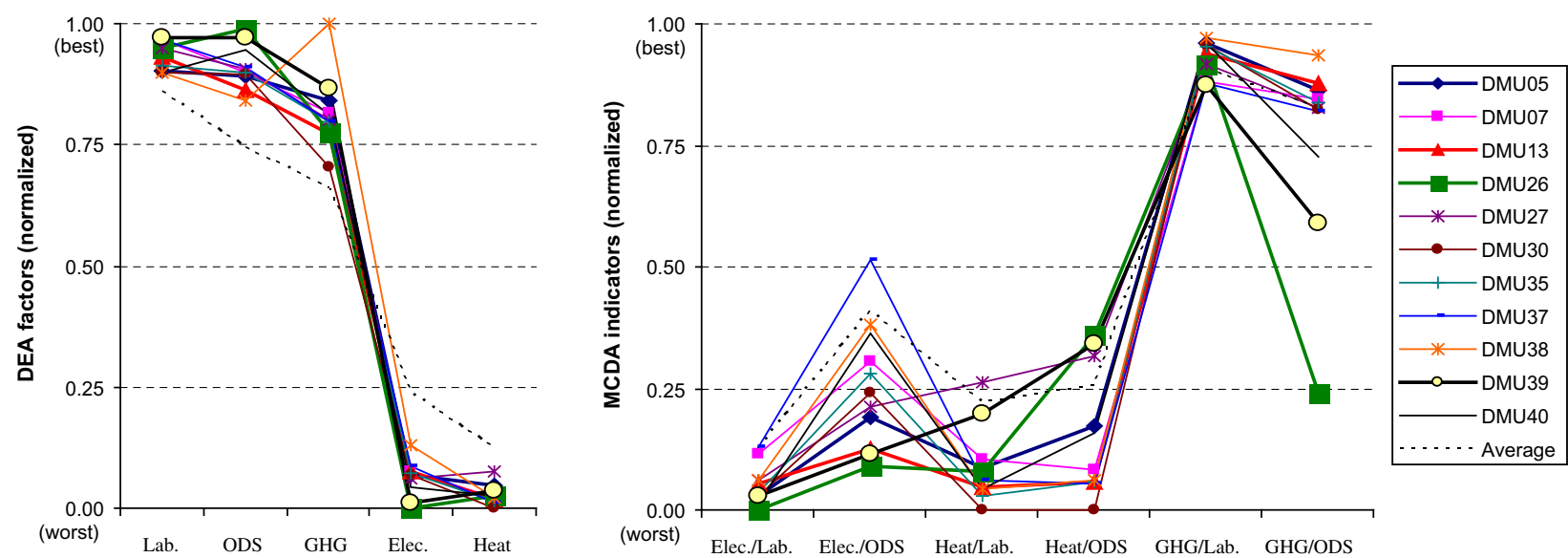

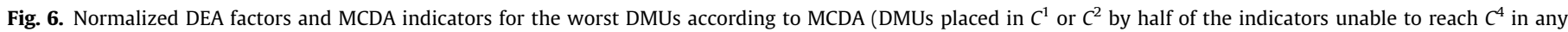
indicator).

26, and 39, shown in thicker lines in both figures). The main exceptions in the correspondence of the two analyses are DMUs 37 and 38 (Fig. 6), which show a higher efficiency than the worst-performing DMUs according to DEA and different profiles: DMU 37 is noticeably ranking better on ratio $g_{2}$ (ELP/ODS); DMU 38 is rather good (almost the best) in terms of factor $o_{3}$ (GHG). Despite these advantages, DMUs 37 and 38 are among the worst-performing DMUs according to MCDA, according to the criterion defined above. On the other hand, DMUs 14 and 33 have very low efficiency according to DEA but are not considered among the worst by MCDA: DMU 14, because it reaches $C^{3}$ on half of the indicators; DMU 33, because it reaches $C^{4}$ on indicator $g_{4}$ (HEA/ODS).

Considering now the DMUs that appear as the most efficient ones in each approach, there are more pronounced differences. Fig. 7 depicts the profiles of the DMUs with the best performance according to DEA, the efficient ones; Fig. 8 depicts the profiles of the best-performing DMUs according to MCDA which we have defined to be those that reach $C^{4}$ on half of the indicators (at least) and are not placed in $C^{1}$ by any indicator (i.e., "pessimistic" classification is $C^{2}$ or better).

The set of efficient DMUs (Fig. 7) presents quite different profiles, but it can be noticed that each of these DMUs excels on one of the 6 MCDA indicators. On the other hand, the efficient DMUs 12 and 17 have a very bad performance judged by two of the factors. Two of the efficient DMUs are also among the best-performing according to MCDA (DMUs 15 and 20, shown in thicker lines in both figures). The remaining efficient DMUs are not considered to be among the best according to MCDA, for being classified in $C^{1}$ by one (or two) of the MCDA indicators: DMU 12 is penalized by its low production of heat per ODS or per labor unit; DMUs 17,18 , and 28 are penalized by being among the 10 that produce more GHG per labor unit. When considering the best DMUs on MCDA (Fig. 8), the least efficient one is DMU 36 (with a relatively high efficiency score of 0.89 ).

The main reason for the differences between the DEA and MCDA analyses is, obviously, the mathematics behind each of these approaches, reflecting different philosophies. The best DMUs according to DEA are the ones for which we cannot observe an objectively better DMU (or a combination of DMUs). The best DMUs according to MCDA are the ones attaining the highest categories in a given number of indicators representing partial productivity measures (for this comparison we considered half of the indicators, although a higher majority level could have been required) and that do not have any weaknesses.

Some differences between the two analyses can also be attributed to different degrees of discrimination. For instance, in MCDA when we defined a lower bound of 2300 to be considered as "Very good" for the indicator electricity/labor, a DMU with a ratio of 2300 is treated the same way as a DMU with a ratio of 3000. In DEA, this change would probably have a high impact. The difference between the approaches can be diminished as the number of categories increases, as the discrimination among DMUs would increase in the MCDA analysis. Another source of differences is the choice of indicators $g_{5}$ and $g_{6}$ instead of electricity/GHG $\left(o_{1} / o_{3}\right)$ and 

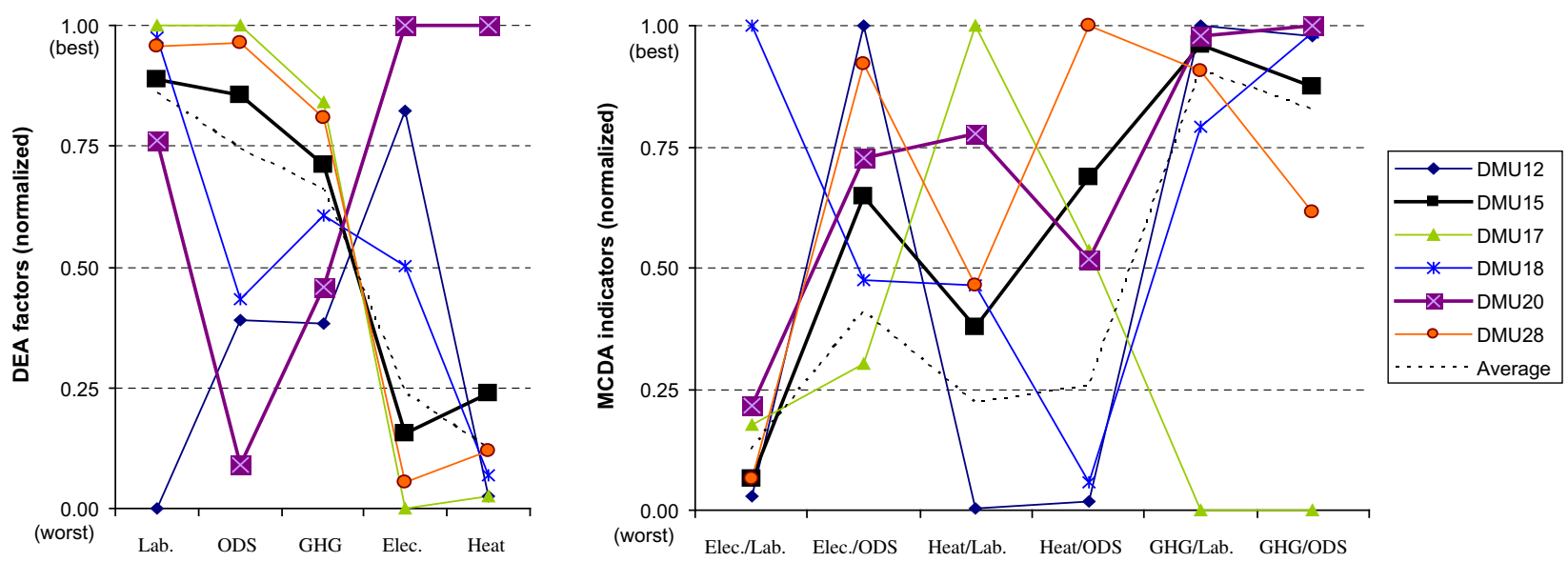

Fig. 7. Normalized DEA factors and MCDA indicators for the best DMUs according to DEA (efficient DMUs).
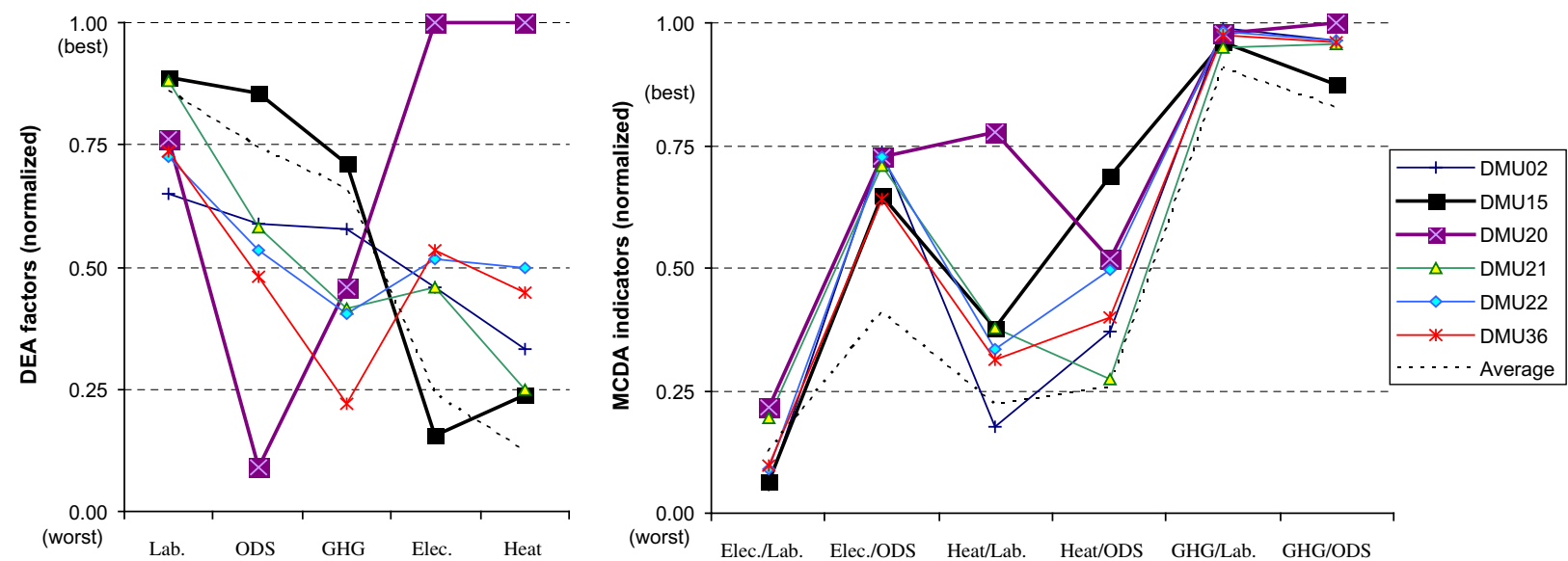

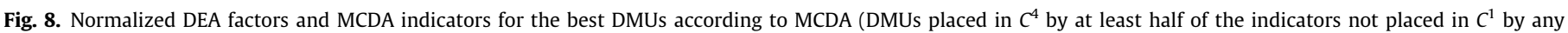
indicator).

heat/GHG $\left(\mathrm{O}_{2} / \mathrm{O}_{3}\right)$, respectively, which are closer to the option of considering GHG as an input in DEA. The results would change, but not radically: the set of the worst DMUs on MCDA would include the same ones plus the DMUs 37, 38, and 39; the set of the best DMUs on MCDA would also include the present ones, plus DMU 18. However, the intention of the MCDA study was not to try to obtain results as close as possible to DEA results, but to evaluate the DMUs independently of the DEA results.

\subsection{Further analyses with IRIS}

The choice of the best and worst DMUs in the MCDA study was performed without making any distinction between the indicators. ELECTRE TRI/IRIS allows to incorporate the managerial judgment about how important each indicator is and whether a very low performance in some indicators may be an impediment to reach the highest categories. Rather than demanding precise numerical weights, the IRIS software can compute the range of categories for a DMU that is compatible with a set of parameter constraints. Let us consider, for instance, the following constraints as an illustrative case:

(a) The evaluator states that the most important output is Electricity, followed by GHG emissions (to be minimized), and lastly by Heat. This implies that the importance of $g_{1}$ (electricity/labor) cannot be lower than the importance of $g_{5}$ (GHG/labor), which in turn cannot be lower than the impor- tance of $g_{3}$ (heat/labor). Analogously, the importance of $g_{2}$ (electricity/ODS) cannot be lower than the importance of $g_{6}$ (GHG/ODS), which in turn cannot be lower than the importance of $g_{4}$ (heat/ODS).

(b) Concerning the inputs, the evaluator states that ODS is more important than labor. This implies that the importance of $g_{2}$ (electricity/ODS) cannot be lower than the importance of $g_{1}$ (electricity/labor). Analogously, the importance of $g_{4}$ (heat/ ODS) cannot be lower than the importance of $g_{3}$ (heat/labor), and the importance of $g_{6}$ (GHG/ODS) cannot be lower than the importance of $g_{5}$ (GHG/labor).

The results corresponding to these requirements are shown in Table 4 . The column "IRIS suggestion" indicates the classification corresponding to the weight values inferred by IRIS ( $k_{1}=k_{6}=0.2$, $\left.k_{2}=0.2667, k_{3}=0.0667, k_{4}=k_{5}=0.1333\right)$. The column "Optimistic" corresponds to a situation where the DMUs could choose their weights (provided that the imposed constraints were satisfied). Optionally, the ELECTRE TRI models also allow incorporating veto thresholds, such that, for instance, a DMU that is classified as $C^{1}$ according to a given indicator will not be able to reach category $C^{4}$ in a multi-criteria evaluation. Supposing the evaluator would consider that the indicators concerning electricity production should have veto power, such that a DMU with a ratio of less than 1100 on $g_{1}$ (electricity/labor) or less than 1130 on $g_{2}$ (electricity/ ODS), i.e., DMUs unable to reach $C^{3}$ on these indicators, could not 
Table 4

Classification subject to importance constraints

\begin{tabular}{llllll}
\hline DMU & IRIS suggestion & Optimistic & DMU & IRIS suggestion & Optimistic \\
\hline 1 & 3 & 3 & 22 & 4 & 4 \\
2 & 4 & 4 & 23 & 3 & 3 \\
3 & 3 & 3 & 24 & 2 & 2 \\
4 & 2 & 3 & 25 & 1 & 1 \\
5 & 1 & 2 & 26 & 1 & 1 \\
6 & 3 & 3 & 27 & 2 & 2 \\
7 & 2 & 28 & 2 & 4 \\
8 & 2 & 2 & 1 & 4 \\
9 & 3 & 3 & 30 & 1 & 1 \\
10 & 3 & 3 & 2 & 3 \\
11 & 3 & 3 & 31 & 2 & 1 \\
12 & 4 & 3 & 32 & 1 & 3 \\
13 & 2 & 4 & 33 & 1 & 2 \\
14 & 2 & 2 & 34 & 3 & 4 \\
15 & 3 & 3 & 35 & 2 & 3 \\
16 & 3 & 4 & 36 & 3 & 2 \\
17 & 2 & 3 & 37 & 2 & 1 \\
18 & 3 & 2 & 38 & 2 & 2 \\
19 & 3 & 4 & 39 & 1 & 4 \\
20 & 4 & 4 & 40 & 1 & \\
21 & 3 & 4 & 41 & 4 & \\
\hline
\end{tabular}

achieve category $C^{4}$, then DMUs $2,12,15,28$, and 29 could no longer reach the best category on the "optimistic" sorting.

Similar types of ad hoc robustness analysis could easily be carried out in order to capture the imprecision associated with the specification of some parameters and identify those for which small changes reveal to have a substantial impact on the results. A form which is easily perceived by managers is to ask for intervals for some of the parameters (for instance, the weights), aimed at capturing information that is not precisely known but can be taken as bounded within some acceptable limits.

When taking the suggested parameters inferred by IRIS, taking into account the constraints imposed on the indicator (criterion) weights, the results may be rather different from those obtained with DEA. However, the MCDA analysis may complement the DEA analysis by providing another perspective from which the conclusions of DEA may be either strengthened or weakened.

\section{Discussion and conclusions}

DEA is a data-oriented approach that requires no a priori specification of the functional form of the production model converting inputs into outputs. Units are then free to choose their most favorable weights for becoming efficient when compared with their peers. On the other hand, this can present a disadvantage whenever over-specialization must be avoided in the consumption of inputs or the production of outputs, which amounts to practically ignore some inputs and outputs. Moreover, managerial preference information is often required, since inputs and outputs do not generally have the same importance in assessing the efficiency of operational units. Therefore, models for efficiency evaluation must explicitly incorporate meaningful techniques to take weights into account, understood as coefficients of relative importance of inputs and outputs. This has been the main motivation for the use of MCDA techniques, in order to assess the extent by which these could overcome those characteristics of DEA, and what adaptations would be needed to improve the quality of the assessment.

We have chosen to frame the MCDA as a sorting problem, which requires that the inputs and outputs are used in the form of efficiency criteria, either combining all the possible indicators (as in this study's example), or selecting the most representative ones for the analyst, or by using a common denominator that can act as a proxy for the scale of operation. By framing the MCDA as a sorting problem, the analyst may enrich the DEA results by being able to separate the DMUs into meaningful categories, possibly taking into account the existence of absolute standards. For instance, an authority certifying sustainable development practices may use this type of MCDA to label energy production plants according to their efficiency, taking into account the inputs they consume, the energy and other desirable outputs they produce, as well as greenhouse gas emissions and other undesirable outputs. Additionally, MCDA facilitates the definition of meaningful constraints on the weights. The MCDA analysis may also be used when not all DMUs are known, e.g., for a case-by-case evaluation of projected DMUs as they are submitted over a period of time.

The IRIS sorting tool used in this study allows incorporating managerial information (weights, category bounds and possibly veto thresholds) easily, yet not demanding precise values for the weights. The results obtained with IRIS enable a rich analysis of the DMUs: a conjunctive type of analysis where the weakest indicator of each DMU determines the result, or a disjunctive type of analysis where each DMU can choose the indicator that is more favorable to its evaluation, or an intermediate majority may be required, or the proposal of IRIS may be used (based on weights inference).

The ranking provided by DEA appears to be more discriminative, but the use of DEA to produce rankings has been criticized (Bouyssou, 1999). Nevertheless, DEA models such as CCR and BCC yield performance measures that can be communicated to managers as indicating by how much outputs have to increase (or inputs to decrease) to achieve efficiency. More importantly, it allows indicating for each inefficient DMU a set of efficient peers against which the DMU should compare itself as a benchmark for improvement. Therefore, we are not proposing MCDA as an approach to replace DEA as a performance evaluation tool. DEA and MCDA should rather be used as complementary techniques, namely as far as the meaningful introduction of managerial preferences is concerned.

In a combined use of MCDA and DEA for evaluating biogas plants, IRIS can be particularly suited to attribute efficiency labels (corresponding to the categories) that take into account the importance that the evaluator (e.g., a regulator) attaches to each indicator, as well as possibly some veto thresholds to prevent a plant that is particularly weak at some indicator to achieve a high category. DEA, on the other hand, can be particularly suited to identify DMUs with efficiency gaps relative to the state of the art, given the observed efficiency frontier. Knowing what can be done (DEA), and what should be done (MCDA), would then help plant managers to keep improving their operation.

\section{Acknowledgements}

This research has been partly funded by the Austrian Federal Ministry of Transport, Innovation, and Technology (BMVIT) under Project Grant No. 807742, and the Portuguese Foundation for Science and Technology under Project Grants POCTI/ESE/38422/ 2001, POCI/EGE/58371/2004 and PTDC/ENR/64971/2006. Fruitful comments provided by three anonymous referees, and Michael Laaber of IFA-Tulln, are gratefully acknowledged.

\section{References}

Abbasi, S.A., Abbasi, N., 2000. The likely adverse environmental impacts of renewable energy sources. Applied Energy 65 (1-4), 121-144.

Banker, R.D., Charnes, A., Cooper, W.W., 1984. Some models for estimating technical and scale inefficiencies in data envelopment analysis. Management Science 30 (9), 1078-1092. 
Bouyssou, D., 1999. Using DEA as a tool for MCDM: Some remarks. Journal of the Operational Research Society 50 (9), 974-978.

Braschkat, J., Gaertner, S.O., Reinhart, G.A., 2003. Biogas versus other fuels: A comparative environmental assessment. In: The Future of Biogas in Europe II. Esbjerg. <http://websvr5.sdu.dk/bio/konf.rapport.pdf>.

Braun, R., Madlener, R., Laaber, M. 2005. Efficiency evaluation of energy crop digestion plants. In: Seventh FAO/SREN Workshop on "The Future of Biogas for Sustainable Energy Production in Europe”, Uppsala, Sweden, December 2005.

Braun, R., Laaber, M., Madlener, R., 2007. Aufbau eines Bewertungssystems für Biogasanlagen - "Gütesiegel Biogas" Endbericht, Projekt im Auftrag des BMVIT (Forschungsprogramm “Eneregiesysteme der Zukunft"), Tulln, Mai (in German).

Charnes, A., Cooper, W.W., Rhodes, E., 1978. Measuring the efficiency of decision making units. European Journal of Operational Research 2 (6), 429-444.

Charnes, A., Cooper, W.W., Golany, B., Seiford, L., Stutz, J., 1985. Foundations of data envelopment analysis for Pareto-Koopmans efficient empirical production functions. Journal of Econometrics 30 (1-2), 91-107.

Cooper, W.W., Seiford, L.M., Tone, K., 2000. Data Envelopment Analysis: A Comprehensive Text with Models, Applications, References and DEA-Solver Software. Kluwer Academic Publishers, Boston/Dordrecht/London.

Cooper, W.W., Seiford, L.M., Zhu, J. (Eds.), 2004. Handbook on Data Envelopment Analysis. Kluwer Academic Publishers.

Dias, L., Mousseau, V., 2003. IRIS - Interactive Robustness Analysis and Parameters Interference for Multicriteria Sorting Problems (Version 2.0), INESC, Coimbra.

Dias, L., Mousseau, V., Figureira, J., Clímaco, J., 2002. An aggregation/disaggregation approach to obtain robust conclusions with ELECTRE TRI. European Journal of Operational Research 138 (2), 332-348.

Dincer, I., Rosen, M.A., 1999. Energy, environment and sustainable development Applied Energy 64 (1-4), 427-440.

Dyson, R.G., Allen, R., Camanho, A.S., Podinovski, V.V., Sarrico, C.S., Shale, E.A., 2001 Pitfalls and protocols in DEA. European Journal of Operational Research 132 (2), 245-259.

Energie-Control, 2006. Bericht über die Ökostrom-Entwicklung und fossile KraftWärme-Kopplung in Österreich. Energie-Control GmbH, Wien (in German).

Green Electricity Act, 2002. Bundesgesetz, mit dem Neuregelungen auf dem Gebiet der Elektrizitätserzeugung aus erneuerbaren Energieträgern und auf dem Gebiet der Wärme-Kraft-Kopplung erlassen werden (Ökostromgesetz), BGBl. No. 149/2002 (accompanying ordinance: BGBl. II No. 508/2002), Vienna.

Joro, T., Viitala, E.J., 2004. Weight-restricted DEA in action: From expert opinions to mathematical models. Journal of the Operational Research Society 55 (8), 814-821.
Laaber, M., Kirchmayr, R., Madlener, R., Braun, R., 2005. Development of an evaluation system for biogas plants. In: Ahring, B.K., Hartmann, H. (Eds.), Proceedings of the 4th International Symposium on Anaerobic Digestion of Solid Waste (ADSW 2005), 31 August-2 September 2005, Copenhagen, Denmark.

Lovell, C.A.K., Pastor, J., 1995. Units invariant and translation invariant DEA models. Operations Research Letters 18 (3), 147-151.

Madlener, R., 2006. Productivity Benchmarking with Data Envelopment Analysis: Towards an Austrian Quality Standard for Biogas CHP Plants. Paper presented at the 29th IAEE International Conference, Potsdam, Germany, 7-10 June 2006.

Madlener, R., Honegger, A., 2006. Productivity Benchmarking with Data Envelopment Analysis: Guidance for an Austrian Quality Standard for Biogas Cogeneration Plants, CEPE Report No. 7, Centre for Energy Policy and Economics (CEPE), ETH Zurich, December.

Mousseau, V., Slowinski, R., Zielniewicz, P., 2000. A user-oriented implementation of the ELECTRE-TRI method integrating preference elicitation support. Computers \& Operations Research 27 (7-8), 757-777.

Podinovski, V.V., 2004. Production trade-offs and weight restrictions in data envelopment analysis. Journal of the Operational Research Society 55 (12), 1311-1322.

Roy, B., 1996. Multicriteria Methodology for Decision Analysis. Kluwer Academic Publishers, Dordrecht.

Roy, B., 1998. A missing link in OR-DA: Robustness analysis. Foundations of Computing and Decision Sciences 23 (3), 141-160.

Scheel, H., 2001. Undesirable outputs in efficiency valuations. European Journal of Operational Research 132 (2), 400-410.

Seiford, L.M., Zhu, J., 2002. Modeling undesirable factors in efficiency evaluation. European Journal of Operational Research 142 (1), 16-20.

Thanassoulis, E., Portela, M.C., Allen, R., 2004. Incorporating value judgments in DEA. In: Cooper, W.W., Seiford, L.M., Zhu, J. (Eds.), Handbook on Data Envelopment Analysis. Kluwer, Boston, pp. 99-137.

Vincke, P., 1999. Robust solutions and methods in decision-aid. Journal of Multicriteria Decision Analysis 8 (3), 181-187.

Walla, C., 2005. Wirtschaftlichkeit von Biogasanlagen. Dissertation, Department für Wirtschafts- und Sozialwissenschaften, Institut für Agrar- und Forstökonomie, Universität für Bodenkultur Wien, Wien, Februar (in German).

Yu, W., 1992. ELECTRE TRI: Aspects méthodologiques et manuel d'utilisation. Document du LAMSADE 80, Université Paris-Dauphine. 